\title{
Influência sociodemográfica na qualidade de vida relacionada com a saúde dos adolescentes
}

Sociodemographic influence in health-related quality of life in adolescents Influencia sociodemográfica en la calidad de vida relacionada con la salud de los adolescentes

Isabel Bica iD https://orcid.org/0000-0002-7019-0132

Luís Miguel Duarte Pinho iD https://orcid.org/0000-0001-6888-1340² Ernestina Maria Batoca Silva id https://orcid.org/0000-0002-4401-6296

Graça Aparício id https://orcid.org/0000-0001-6149-062x'

João Duarte io https://orcid.org/0000-0002-2390-3910 ${ }^{1}$

José Costa io https://orcid.org/0000-0001-6441-6177

Carlos Albuquerque io https://orcid.org/0000-0002-2297-0636 ${ }^{1}$

\section{Como citar:}

Bica I, Pinho LM, Silva EM, Aparício G, Duarte $\mathrm{J}$, Costa J, et al. Influência sociodemográfica

na qualidade de vida relacionada com a saúde dos adolescentes. Acta Paul Enferm. 2020;33:e-APE20190054

DOI

http://dx.doi.org/10.37689/ acta-ape/2020A00054

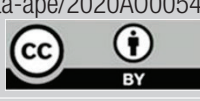

Descritores

Saúde do adolescente; Qualidade de vida; Perceção

Keywords

Adolescent health; Quality of life; Perception

Descriptores

Salud del adolescente; Calidad de vida; Percepción

Submetido 9 de Março de 2019

Aceito

22 de Julho de 2019

\section{Autor correspondente}

Isabel Bica

Rua Dom João Crisóstomo Gomes de Almeida, 102, 3500-843, Viseu, Portugal. https://orcid.org/0000-0003-1439-0372 E-mail: isabelbica@gmail.com

\section{Resumo}

Objetivo: Identificar as variáveis sociodemográficas (sexo, idade, escolaridade e coabitação) que influenciam a perceção da qualidade de vida relacionada com a saúde dos adolescentes.

Métodos: Estudo descritivo-correlacional de natureza quantitativa, numa amostra não probabilística por conveniência, constituída por 567 adolescentes, a frequentar $02^{\circ}$ e $3^{\circ}$ Ciclo do Ensino Básico num Agrupamento de Escolas do centro de Portugal. Os participantes apresentavam média de idade de 12,4 anos ( $D p=1,59)$, os rapazes entre 9-16 anos, e as meninas entre 10-17 anos, 50.6\% do sexo feminino e a maioria coabita numa família nuclear $(77,4 \%)$. Na coleta de dados utilizou-se a versão portuguesa da escala Kidscreen-52(1) e questões de caracterização sociodemográfica. Os dados foram coletados no período de janeiro a julho de 2018 e o tratamento estatístico foi realizado utilizando o Statistical Package for the Social Sciences ${ }^{\circledR}$ (SPSS - versão 24.0).

Resultados: Os adolescentes têm uma perceção positiva da sua qualidade de vida relacionada com a saúde, com diferença estatística para a idade, teste Anova ( $F=31,980 ; p=0.000)$, para 0 ano de escolaridade, ( $F=15,293 ; p=0.000)$ e Coabitação ( $F=11,491 ; p=0.010)$.

Conclusão: Os rapazes apresentam uma melhor percepção sobre a qualidade de vida, assim como os adolescentes mais jovens e os que coabitam com os pais (mãe e pai).

\section{Abstract}

Objective: To determine the sociodemographic variables (gender, age, level of formal education and cohabitation) that have influenced the perception of health-related quality of life of adolescents.

Methods: This was a descriptive, correlational and quantitative study including a non-probabilistic convenience sample of 567 adolescents. Of these, 50.6\% were girls and attended 2nd and 3rd cycles of basic education in grouping of schools in central Portugal. The participants mean age was 12.4 years-old ( $S D=1.59$ ), boys age ranged from 9 to 16 years-old and girls from 10 to 17 , most of them lived within a nuclear family $(77.4 \%)$. Data were collected from January to June 2018 using a Portuguese version of Kidscreen-52(1) scale along with a questionnaire of sociodemographic characterization. The IBM SPSS Statistics for Windows (Version 24.0. Armonk, NY: IBM Corp) was used for statistical analyses.

Results: Adolescents have a positive perception of their quality of life regarding health. A statistical difference related with age, One-way ANOVA test $(F=31.980 ; p=0.000)$, school year $(F=15.293 ; p=0.000)$ and cohabitation ( $F=11.491 ; p=0.010)$.

Conclusion: Boys present a higher perception of quality of life, as well as younger adolescents and those who live with their parents (mother and father). 


\section{Resumen}

Objetivo: Identificar las variables sociodemográficas (sexo, edad, escolaridad y cohabitación) que influyen en la percepción de calidad de vida relacionada con la salud de los adolescentes.

Métodos: Estudio descriptivo correlacional de naturaleza cuantitativa, en un muestreo no probabilístico por conveniencia, constituido por 567 adolescentes que cursan de $5^{\circ}$ a $9^{\circ}$ año de primaria en un Agrupamiento de Escuelas del centro de Portugal. Los participantes tenían edad promedio de 12,4 años $(\mathrm{D} p=1,59)$, los varones de 9 a 16 años y las mujeres de 10 a 17 años, 50,6\% de sexo femenino y la mayoría cohabita en una familia nuclear (77,4\%). En la recolección de datos se utilizó la versión portuguesa de la escala Kidscreen-52 $2^{(1)}$ y cuestiones de caracterización sociodemográfica. Los datos se recolectaron de enero a julio de 2018 y el tratamiento estadístico se realizó utilizando el Statistical Package for the Social Sciences® (SPSS - versión 24.0).

Resultados: Los adolescentes tienen una percepción positiva de su calidad de vida relacionada con la salud, con diferencia estadística por edad, test Anova ( $F=31,980 ; p=0.000)$, por año de escolaridad, $(F=15,293 ; p=0.000)$ y cohabitación ( $F=11,491 ; p=0.010)$.

Conclusión: Los varones presentan una mejor percepción sobre la calidad de vida, así como los adolescentes más jóvenes y los que cohabitan con los padres (madre y padre).

\section{Introdução}

A qualidade de vida é uma preocupação da sociedade contemporânea. Existe, por isso, um interesse crescente sobre o bem-estar das pessoas. Neste contexto, considerando os adolescentes como seres em constante desenvolvimento, eles necessitam de crescer de forma equilibrada e saudável no seio de um ambiente seguro e estruturado, que lhes permita um desenvolvimento positivo a nível físico, educativo, emocional e social. Para a Organização Mundial de Saúde ${ }^{(1,2)}$ e Direção Geral da Saúde de Portugal, ${ }^{(3,4)}$ promover o desenvolvimento saudável dos adolescentes é considerado um investimento no futuro, uma vez que as opçóes saudáveis produzem resultados no bem-estar nessa etapa da vida mas igualmente na vida adulta. É na adolescência que se adquire consistência para os alicerces da saúde futura, os estilos de vida, as atitudes e os padróes de comportamento que podem garantir uma melhor qualidade de vida.

O conceito de Qualidade de Vida (QV) encontra-se relacionado com a perceção que o indivíduo possui da sua posição na vida, no contexto do sistema cultural e de valores em que as pessoas vivem, dos seus objetivos e das suas expetativas. Por isso, é um conceito amplo que é afetado de forma complexa pela saúde física da pessoa, pelo seu estado psicológico, nível de dependência, relaçóes sociais, crenças e convicçôes pessoais e pelo ambiente em que vive. ${ }^{(5)}$

A qualidade de vida relacionada à saúde (QVRS) tem no seu conceito indicadores que direcionam a saúde percebida, portanto, segundo Gaspar \&

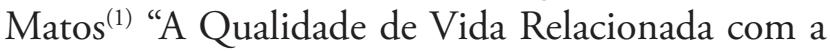

Saúde é considerada como um constructo que engloba componentes do bem-estar e funções físicas, emocionais, mentais, sociais e comportamentais, como são percebidos pelos próprios e pelos outros".

$\mathrm{O}$ estudo do desenvolvimento da criança e do adolescente e da qualidade de vida relacionada com a saúde, cada vez mais, dá atenção à interação de múltiplas variáveis contextuais na vida do adolescente em desenvolvimento, pois, uma vez identificados os fatores de risco, eles podem vir a ser o ponto de entrada ou o foco para as estratégias e açôes de promoção da saúde e prevenção de doenças. ${ }^{(6)}$ Por outro lado perspetivar a saúde dos adolescentes a partir de uma perspetiva de género tem um potencial considerável para a definição de estratégias que reduzam os diferenciais na saúde associados ao género e a sua consequente persistência na vida adulta. Segundo alguns autores ${ }^{(7)}$ as diferenças associadas ao género em alguns comportamentos de saúde tendem a aumentar durante a adolescência.

A área da qualidade de vida é uma área de investigação transversal a várias áreas do conhecimento, nomeadamente no âmbito das ciências sociais e humanas. Porém, no campo da saúde, a QV assume particular relevância para a enfermagem, pois o seu foco de atenção está centrado na pessoa/família e não no corpo com problema de saúde.

Uma vez que os estudos realizados sobre a qualidade de vida relacionada com a saúde em crianças e adolescentes ainda são escassos e a adolescência é um importante marco no desenvolvimento humano, esta investigação pretender contribuir para uma melhor compreensão do impacto dos fatores sociodemográficos na qualidade de vida de um grupo de adolescentes, partindo-se do questionamento: 
Que variáveis sociodemográficas (sexo, idade, escolaridade, coabitação) influenciam a perceção da qualidade de vida relacionada com a saúde dos adolescentes?

Neste contexto, este estudo tem como objetivo identificar as variáveis sociodemográficas (sexo, idade, escolaridade e coabitação) que influenciam a perceção da qualidade de vida relacionada com a saúde dos adolescentes.

\section{Métodos}

Estudo descritivo, transversal e de natureza quantitativa, numa amostra não probabilística por conveniência, constituída por 567 adolescentes, sendo $50.6 \%$ do sexo feminino, com idades compreendidas entre os 10 e os 17 anos (Média= 12,4; $\mathrm{DP}=1,59$ anos) e uma média de idade de 12,38 anos para as meninas e 12,41 anos para os rapazes. Frequentam escolas do $2^{\circ}$ e $3^{\circ}$ ciclo do Ensino Básico de um Agrupamento de Escolas da região centro de Portugal. A maioria $(77,4 \%)$ dos adolescentes coabita com ambos os pais, $18,5 \%$ com a mãe e $4,1 \%$ com outros (avós, pai, tios).

A pesquisa teve início após o parecer favorável da Comissão de Ética da Escola Superior de Saúde de Viseu (Parecer no 20) e da Comissão Nacional de Proteção de Dados (Ref 03.01, Ofício 38790 de 18/12/2017), autorização do Ministério da Educação com registo No 0012100017 , autorização do Director do Agrupamento de Escolas e o Termo Consentimento Livre e Esclarecido, assinado pelos encarregados de educação, decorrendo no período de janeiro a julho de 2018 .

O estudo insere-se no projecto "MAISaúdeMental - Monitorização e Avaliação dos Indicadores de Saúde Mental das crianças e adolescentes: da investigação à prática”, com a identificação 023293 CENTRO-01-0145-FEDER-023293 financiado pelo Programa "Portugal 2020" e a decorrer na Escola Superior de Saúde de Viseu, Portugal.

$\mathrm{O}$ instrumento utilizado na coleta de dados foi a Escala de qualidade de vida relacionada com a saúde - KIDSCREEN 52, versão portuguesa, adaptada e validada pela equipa do projeto "Aventura Social ". (1,6)
Esta é constituída 52 itens e dez dimensóes que descrevem a Qualidade de vida relacionada com a saúde (QVRS): Saúde e Atividade Física; Sentimentos; Estado de Humor Geral; Auto-percepção (sobre ti próprio); Tempo Livre; Família, Ambiente Familiar e Vizinhança; Questóes Económicas; Amigos; Ambiente Escolar e Aprendizagem; e Provocação (Bullying). Nas dimensóes, cada item é pontuado numa escala tipo Likert que varia entre 1 = NuncalNada, 2 = Raramente/Pouco; 3 = Algumas vezes/Moderadamente; 4 = Frequentemente/Muito; 5 = Sempre/Totalmente, sendo que quanto mais elevada for a pontuação, maior a perceção da qualidade de vida pelas crianças e adolescentes. A dimensão "Provocação (Bullying)" a pontuaçáo foi invertida, apontando valores elevados na qualidade de vida se os sentimentos negativos forem raros. Uma pontuação baixa implica sentir-se atormentado, provocado e rejeitado pelos seus pares e uma pontuação elevada revela não se sentir provocado, sentir-se respeitado e aceite pelos pares.

Após somatório dos diferentes itens pertencentes a cada dimensão, os resultados foram transformados em valores de 0 a 100 através da seguinte fórmula (score bruto- score mínimo esperado) /amplitude) * 100 .

Os resultados foram agrupados por dimensóes e apurou-se que um score de 50 é o valor médio da cada dimensão. Desse modo, considerou-se que a perceção das crianças/adolescentes sobre a qualidade de vida relacionada com a saúde foi positiva quando apresentou scores superiores a esse valor médio e, pelo contrário, scores inferiores a esse valor médio representaram uma perceçáo negativa da qualidade de vida relacionada com a saúde.

A informação recolhida foi armazenada e posteriormente processada e analisada utilizando o Statistical Package for the Social Sciences (SPSS - versão 24.0), com base na análise estatística descritiva e analítica, com recurso ao Teste-t para diferença de médias, Teste de Levene, Teste One-Way ANOVA e Kruskal-Wallis, utilizando-se o Intervalo de Confiança (IC) a 95\% (significância p<0,05).

\section{Resultados}

Analisou-se a relação entre a QVRS e as variáveis sociodemográficas (sexo, idade, escolaridade e coa- 
bitação). No intuito de verificar se a QVRS e as dimensóes que a constituem eram discriminadas pelo sexo efetuou-se um teste-t para diferença de médias. No global não se apurou diferença estatística significativa na perceção da QVRS entre rapazes e meninas, contudo, nas diferentes dimensões observou-se que as variâncias não são homogéneas para as dimensóes Sentimentos, Familia, ambiente familiar e vizinhança, Questóes económicas, Amigos/ as e Ambiente escolar e aprendizagem e pelos índices médios observou-se que os rapazes apresentam melhor perceção de QVRS exceto no que respeita às Questóes económicas, Ambiente escolar e aprendizagem e Provocação, relativamente às meninas. Os valores de $\mathrm{t}$ indicam que existem diferenças significativas para as dimensóes Saúde e atividade física, Estado de humor geral, Autoestima, Autonomia e Ambiente escolar e aprendizagem, sendo apenas este último superior e significativo nas jovens $(\mathrm{p}<0.05)$ (Tabela 1$)$.

Ao analisar a influência da idade na QVRS e nas diferentes dimensóes, verificou-se (Tabela 2), existir significância estatística entre a idade e a QVRS, sendo os valores médios superiores em todas as dimensôes na faixa etária 10-11 anos, exceto na dimensão Provocação, cuja média foi mais elevada nos adolescentes ( $\geq 14$ anos), porém, sem significância estatística ( $p>0.05)$. Pelos resultados expostos, infere-se que ocorre uma perceção menos positiva da QVRS à medida que aumenta a idade em todas as dimensóes, com exceção da dimensão Provocação (Bullying).

Indagando a influência da escolaridade na QVRS e nas suas diferentes dimensóes observou-se que o ano de escolaridade influencia significativamente a perceção de QVRS em todas as dimensões $(\mathrm{p}<0.05)$, com índices médios mais elevados nas crianças do $5^{\circ}$ ano e $6^{\circ}$ de escolaridade, exceto na dimensão Provocação, onde se destacam os adolescentes do $8^{\circ}$ ano de escolaridade (Tabela 3 ).

\section{Relação entre a coabitação e QVRS}

A analise da influência da família (pessoas com quem coabitam) sobre a perceção da QVRS dos adolescen-

Tabela 1. Teste-t entre o sexo e a QVRS

\begin{tabular}{|c|c|c|c|c|c|c|c|}
\hline \multirow[t]{2}{*}{ Dimensões } & \multicolumn{2}{|c|}{$\begin{array}{l}\text { Masculino } \\
(\mathrm{n}=280)\end{array}$} & \multicolumn{2}{|c|}{$\begin{array}{c}\text { Feminino } \\
(\mathrm{n}=287)\end{array}$} & \multirow{2}{*}{$\begin{array}{c}\text { Teste Levene } \\
\text { p-value }\end{array}$} & \multicolumn{2}{|c|}{ Teste $t$} \\
\hline & Média & Desvio-Padrão & Média & Desvio-Padrão & & $\mathrm{t}$ & $p$-value \\
\hline Saúde e atividade física & 79,4 & 14,8 & 73,7 & 17,5 & 0,008 & 4,230 & 0,000 \\
\hline Sentimentos & 82,4 & 15,1 & 79,8 & 17,1 & 0,129 & 1,928 & 0,054 \\
\hline Estado de humor geral & 80,8 & 16,5 & 77,4 & 19,7 & 0,000 & 2,180 & 0,030 \\
\hline Sobre ti próprio & 62,9 & 12,9 & 59,7 & 14,8 & 0,014 & 2,789 & 0,005 \\
\hline Tempo livre & 63,8 & 14,9 & 60,6 & 17,3 & 0,004 & 2,385 & 0,018 \\
\hline Família, ambiente familiar e vizinhança & 84,1 & 15,5 & 83,3 & 16,4 & 0,461 & 0,576 & 0,565 \\
\hline Questões económicas & 82,6 & 22,7 & 84,9 & 21,0 & 0,106 & $-1,292$ & 0,197 \\
\hline Amigos(as) & 81,8 & 16,3 & 81,6 & 16,0 & 0,914 & 0,154 & 0,878 \\
\hline Ambiente escolar e aprendizagem & 69,5 & 17,6 & 73,0 & 18,8 & 0,338 & $-2,287$ & 0,023 \\
\hline Provocação & 86,8 & 19,5 & 89,4 & 15,6 & 0,019 & $-1,750$ & 0,081 \\
\hline Score Global & 80,2 & 11,4 & 78,68 & 16,6 & 0,002 & 1,432 & 0,153 \\
\hline
\end{tabular}

Tabela 2. Teste One-Way ANOVA entre a Idade e a QVRS

\begin{tabular}{|c|c|c|c|c|c|c|c|c|c|}
\hline \multirow[t]{2}{*}{ Dimensões } & \multicolumn{2}{|c|}{$\begin{array}{c}10-11 \text { anos }(1) \\
(n=190)\end{array}$} & \multicolumn{2}{|c|}{$\begin{array}{c}12-13 \text { anos }(2) \\
(n=218)\end{array}$} & \multicolumn{2}{|c|}{$\begin{array}{c}\geq 14 \text { anos }(3) \\
\quad(n=159)\end{array}$} & \multicolumn{2}{|c|}{ ANOVA } & \multirow[t]{2}{*}{$\operatorname{VE}(\%)$} \\
\hline & Média & DP & Média & DP & Média & DP & $\mathrm{F}$ & $\mathrm{p}$-value & \\
\hline Saúde e atividade física & 80,7 & 15,0 & 77,4 & 16,3 & 70,3 & 16,6 & 19,000 & 0,000 & 6,31 \\
\hline Sentimentos & 86,6 & 14,0 & 80,4 & 16,7 & 75,4 & 16,0 & 22,241 & 0,000 & 7,31 \\
\hline Estado de humor geral & 84,2 & 16,0 & 77,7 & 18,4 & 74,8 & 19,3 & 13,031 & 0,000 & 4,42 \\
\hline Sobre ti próprio & 66,9 & 10,5 & 61,0 & 14,8 & 55,1 & 13,7 & 34,868 & 0,000 & 11,00 \\
\hline Tempo livre & 65,6 & 14,3 & 61,4 & 17,8 & 59,1 & 15,4 & 7,527 & 0,001 & 2,60 \\
\hline Família, ambiente familiar e vizinhança & 88,0 & 13,3 & 82,3 & 17,0 & 80,4 & 16,5 & 11,712 & 0,000 & 3,99 \\
\hline Questões económicas & 86,1 & 22,0 & 84,4 & 21,0 & 80,1 & 22,5 & 3,355 & 0,036 & 1,18 \\
\hline Amigos/as & 85,3 & 15,3 & 82,3 & 15,5 & 76,5 & 16,7 & 13,922 & 0,000 & 4,70 \\
\hline Ambiente escolar e aprendizagem & 81,0 & 15,1 & 69,4 & 18,0 & 62,2 & 16,8 & 57,107 & 0,000 & 16,84 \\
\hline Provocação & 88,8 & 16,3 & 86,8 & 20,0 & 89,0 & 15,6 & 0,949 & 0,388 & 0,34 \\
\hline Score Global & 84,5 & 10,5 & 78,8 & 12,7 & 74,3 & 12,3 & 31,980 & 0,000 & 10,19 \\
\hline
\end{tabular}


Tabela 3. Teste One-Way ANOVA entre 0 ano de escolaridade e a QVRS

\begin{tabular}{|c|c|c|c|c|c|c|c|c|c|c|c|c|c|}
\hline \multirow[t]{2}{*}{ Dimensões } & \multicolumn{2}{|c|}{$\begin{array}{c}5^{\circ} \text { ano }(1) \\
(n=96)\end{array}$} & \multicolumn{2}{|c|}{$\begin{array}{c}6^{\circ} \text { ano }(2) \\
(n=119)\end{array}$} & \multicolumn{2}{|c|}{$\begin{array}{l}7^{\circ} \text { ano }(3) \\
(n=101)\end{array}$} & \multicolumn{2}{|c|}{$\begin{array}{c}8^{\circ} \text { ano }(4) \\
(n=122)\end{array}$} & \multicolumn{2}{|c|}{$\begin{array}{c}9^{\circ} \text { ano }(5) \\
(n=129)\end{array}$} & \multicolumn{2}{|c|}{ Teste Anova } & \multirow[t]{2}{*}{$\begin{array}{l}\text { VE } \\
(\%)\end{array}$} \\
\hline & Média & DP & Média & DP & Média & DP & Média & DP & Média & DP & $\mathrm{F}$ & $p$-value & \\
\hline Saúde e atividade física & 80,8 & 15,2 & 78,4 & 17,0 & 79,0 & 15,5 & 75,3 & 17,0 & 70,5 & 15,4 & 7,574 & 0,000 & 5,12 \\
\hline Sentimentos & 87,5 & 12,6 & 85,1 & 15,7 & 82,4 & 15,0 & 77,5 & 17,4 & 75,0 & 16,1 & 13,076 & 0,000 & 8,51 \\
\hline Estado de humor geral & 84,2 & 16,6 & 82,6 & 15,7 & 80,0 & 17,3 & 74,6 & 20,0 & 75,4 & 19,2 & 6,369 & 0,000 & 4,34 \\
\hline Sobre ti próprio & 66,9 & 11,5 & 65,6 & 12,3 & 61,9 & 14,1 & 58,6 & 14,5 & 55,3 & 13,5 & 15,313 & 0,000 & 9,83 \\
\hline Tempo livre & 65,5 & 15,2 & 65,9 & 14,4 & 61,2 & 18,7 & 60,4 & 16,9 & 58,8 & 14,9 & 4,549 & 0,001 & 3,14 \\
\hline Família, ambiente familiar e vizinhança & 86,7 & 15,1 & 88,4 & 12,6 & 82,2 & 17,1 & 82,9 & 16,3 & 79,1 & 16,8 & 6,603 & 0,000 & 4,49 \\
\hline Questões económicas & 84,2 & 22,9 & 86,0 & 22,2 & 81,9 & 23,2 & 88,0 & 17,7 & 78,7 & 22,4 & 3,477 & 0,008 & 2,42 \\
\hline Amigos(as) & 85,9 & 15,0 & 84,0 & 16,5 & 83,9 & 14,9 & 81,0 & 15,4 & 75,3 & 16,5 & 8,176 & 0,000 & 5,50 \\
\hline Ambiente escolar e aprendizagem & 81,6 & 14,8 & 79,2 & 16,0 & 70,5 & 17,4 & 65,5 & 19,0 & 62,4 & 16,0 & 28,556 & 0,000 & 16,89 \\
\hline Provocação & 87,1 & 18,6 & 90,1 & 16,8 & 86,8 & 19,3 & 86,1 & 20,5 & 89,9 & 12,5 & 1,313 & 0,264 & 0,92 \\
\hline Score Global & 84,3 & 11,0 & 83,5 & 11,3 & 79,7 & 12,3 & 77,0 & 12,7 & 74,0 & 12,3 & 15,293 & 0,000 & 9,82 \\
\hline
\end{tabular}

Tabela 4 .Teste de Kruskal-Wallis entre a família e a QVRS

\begin{tabular}{|c|c|c|c|c|c|c|}
\hline \multirow{2}{*}{ Dimensões } & \multirow{2}{*}{$\begin{array}{c}\text { Pais } \\
(\mathrm{n}=439) \\
\text { Mean } \\
\text { Rank }\end{array}$} & \multirow{2}{*}{$\begin{array}{c}\text { Mãe } \\
(n=105) \\
\text { Mean Rank }\end{array}$} & \multirow{2}{*}{$\begin{array}{c}\begin{array}{c}\text { Pai } \\
(\mathrm{n}=8)\end{array} \\
\text { Mean Rank }\end{array}$} & \multirow{2}{*}{$\begin{array}{c}\begin{array}{c}\text { Outros } \\
(n=15)\end{array} \\
\text { Mean Rank }\end{array}$} & \multicolumn{2}{|c|}{ Teste Kruskal-Wallis } \\
\hline & & & & & $x^{2}$ & $p$-value \\
\hline Saúde e atividade física & 298,6 & 240,7 & 295,1 & 241,6 & 10,696 & 0,013 \\
\hline Sentimentos & 293,2 & 254,9 & 264,0 & 230,1 & 6,496 & 0,090 \\
\hline Estado de humor geral & 297,5 & 239,8 & 282,1 & 197,9 & 14,868 & 0,002 \\
\hline Sobre ti próprio & 292,7 & 255,4 & 284,1 & 228,9 & 6,201 & 0,102 \\
\hline Tempo livre & 292,0 & 254,1 & 264,9 & 268,9 & 4,853 & 0,183 \\
\hline Família, ambiente familiar e vizinhança & 290,4 & 267,7 & 202,8 & 253,7 & 4,264 & 0,234 \\
\hline Questões económicas & 294,2 & 257,1 & 204,3 & 217,0 & 10,091 & 0,018 \\
\hline Amigos(as) & 292,2 & 256,4 & 259,7 & 249,9 & 4,966 & 0,174 \\
\hline Ambiente escolar e aprendizagem & 287,0 & 265,4 & 351,4 & 290,5 & 2,897 & 0,408 \\
\hline Provocação & 291,0 & 270,1 & 243,9 & 198,8 & 7,096 & 0,069 \\
\hline Score Global & 296,3 & 243,1 & 269,3 & 219,2 & 11,441 & 0,010 \\
\hline
\end{tabular}

tes, revelou que o tipo de família influencia significativamente a QVRS dos adolescentes, nomeadamente na Saúde e atividade física, Estado de humor geral e Questóes económicas $(\mathrm{p}<0.01)$, indicando melhor perceção de QVRS naqueles que vivem numa família nuclear (constituída pelo pai e mãe) (Tabela 4).

\section{Discussão}

Segundo a perceção dos adolescentes, no âmbito da avaliação de todos os domínios da escala Kidscreen, a sua qualidade de vida é boa, indo de encontro aos estudos nacionais ${ }^{(1)}$ e mesmo internacionais. ${ }^{(8)}$

No que concerne à relação entre as variáveis sociodemográficas (sexo, idade, escolaridade e coabitação) e à perceção da QVRS dos adolescentes, apurou-se, em primeiro lugar, que os rapazes apresentaram valores médios superiores às meninas. São as meninas, contudo, que apresentaram melhor QVRS no que diz respeito à dimensão Ambiente escolar e aprendizagem, tal como referem alguns autores. ${ }^{(7-9)}$ Esta percepção deve-se, provavelmente, ao maior sucesso escolar por parte das meninas, pois estas "em média têm melhores desempenhos escolares do que os rapazes". ${ }^{(10)}$

Os resultados corroboram, em parte, os observados no estudo de Gaspar \& Matos, ${ }^{(1,8)}$ em que os rapazes apresentavam melhor perceção sobre a própria QVRS na maioria das dimensões, à exceção da dimensão Ambiente escolar e aprendizagem. É de referir que, segundo o estudo das autoras, apenas as dimensóes Questóes económicas e Amigos/as não apresentam diferenças em relação ao sexo. Quanto às dimensóes, Saúde e atividade física, Estado de humor geral, Autoestima $e$ Autonomia, verificou-se que os rapazes apresentam valores médios superiores em relação às meninas. Tal facto tem sido associado às alteraçôes na fase pubertária que provocam frequentemente insatisfação com a imagem corporal nas meninas, influenciando assim o seu bem-estar psicoemocional. ${ }^{(7,11-13)}$ Nas meninas a modificaçôes hormonais características da puberdade ocorre acompanhada de alteração de humor, aumento de peso e de gordura corporal, o 
que modifica abruptamente a imagem corporal, deixando-as mais vulneráveis em um periodo que coincide com aumento da demanda social. ${ }^{(14)}$

Relativamente à idade, tal como nos estudos de Gaspar \& Matos, ${ }^{(1,15)}$ os mais novos ( $\leq 11$ anos) são os que apresentam melhor perceção da QVRS $(M=84.5)$, seguindo-se o grupo etário dos 12-13anos $(\mathrm{M}=78.8)$ e por fim o grupo etário superior ou igual a 14 anos $(\mathrm{M}=74.3)$. Os dados empíricos demonstraram que as médias foram mais elevadas em todas as dimensóes para os adolescentes dos 10 e 11 anos de idade, à exceção da dimensão Provocação em que a média foi mais elevada no grupo etário $\geq 14$ anos. Ou seja, a perceção da QVRS vai diminuindo à medida que a idade aumenta, o que tem sido documentado em diversos estudos realizados, quer no âmbito da qualidade de vida, quer na perceção de competências. ${ }^{(1,7,8,15)}$ Gaspar verificou que as crianças apresentam maiores valores médios de perceção de QVRS em relação aos adolescentes, a exceção da dimensão Provocação. No mesmo sentido, Gaspar e Matos $^{(1)}$ obtiveram diferenças significativas entre o grupo das crianças e o grupo de adolescentes, sendo as crianças que demonstram uma percepção de qualidade de vida superior aos adolescentes. Confirma-se assim que, na linha de Bronfenbrenner, ${ }^{(16)}$ a percepção da qualidade de vida em crianças e adolescentes deve integrar uma perspectiva ecológica do seu desenvolvimento e funcionamento. Da mesma forma, num estudo realizado na Suécia, em 2012, com 600 crianças e adolescentes com idades compreendidas entre 11-16 anos, a perceção de QVRS foi significativamente mais baixa em adolescentes do que em crianças. ${ }^{(17)} \mathrm{A}$ diminuição de bem-estar relacionada à idade pode estar associada com a transiçáo física, cognitiva e social da infância para a vida adulta que os rapazes e meninas enfrentam neste estágio. Essas mudanças podem incluir: mudanças físicas, uma nova aparência física que provoca insatisfação e ansiedade; mudanças cognitivas, novas ferramentas cognitivas que permitem que os adolescentes tenham acesso a pensamentos mais complexos (sobre o mundo em geral e sobre si mesmos e suas circunstâncias particulares), a procura da sua própria identidade e mudanças sociais, novos desafios sociais associados a mudanças de relacionamento com pais e colegas, primeiros amores, primeiras experiências sexuais, entre outras. ${ }^{(15)}$
Também no que diz respeito à dimensão Provocação se verificam diferenças associadas à idade mas no sentido inverso, porém sem significância. Contrariamente, num estudo realizado em onze países europeus com 16210 crianças e adolescentes, com idades compreendidas entre 8 a 18 anos, constatou-se que ser mais jovem, haver baixo nível de escolaridade dos pais, bem como o excesso de peso se associava ao aumento de comportamentos de bullying. ${ }^{(18)}$

É de salientar que a dimensão Família, ambiente familiar e vizinhança é uma das dimensóes com valores médios mais elevados nas crianças mais novas. Diversos autores descrevem que os relacionamentos familiares afetam o bem-estar dos adolescentes e que aqueles que promovem a sua autonomia e partilhem tempo com a família incentivam a um clima familiar positivo para uma melhor QVRS na adolescência. ${ }^{(13,15)}$

Segundo um estudo realizado em Portugal, levado a cabo por Maria et al., ${ }^{(19)}$ com 431 adolescentes de média de idade de 12.8 anos, as dimensóes das relaçôes sociais e pares e as relaçóes familiares e autonomia foram as que obtiveram melhores índices médios, sendo as dimensões bem-estar físico e ambiente escolar e aprendizagem as que obtiveram piores resultados. No que diz respeito às pessoas com quem as crianças e adolescentes habitam, na maioria das dimensóes não foram encontradas diferenças significativas, à exceção da dimensão Saúde e atividade física, Estado de humor geral e Questóes económicas. O presente estudo mostra que as crianças e adolescentes que coabitam numa família nuclear apresentam melhor perceção sobre a própria QVRS, indo de encontro aos resultados do estudo realizado por Berman et al., ${ }^{(17)}$ em que o autor verificou que as crianças e adolescentes que habitavam com ambos os pais apresentavam melhor perceção sobre a própria QVRS.

\section{Conclusão}

No presente estudo apurou-se que os adolescentes do sexo masculino apresentam uma melhor percepção sobre a QVRS comparativamente ao sexo oposto. Os adolescentes mais jovens, os quais frequentam um nível inferior de escolaridade, assim como os que coabitam numa família nuclear tradicional, apresentam 
uma melhor percepção sobre a QVRS. Sabendo do impacto dos fatores sociodemográficos na qualidade de vida relacionada com a saúde dos adolescentes, tornase imperativo a procura de mecanismos ou estratégias de intervenção tanto na comunidade como na escola e na família, adequadas ao género, idade e contexto familiar, para minimizar os efeitos que possam ter no desenvolvimento físico, mental e social das crianças e adolescentes. Estes resultados têm como finalidade, no âmbito do projeto MaiSaúde Mental, contribuir para a elaboração de um programa de intervenção em meio escolar, dirigido às crianças e adolescentes, professores e pais/encarregados de educação.

\section{Agradecimentos}

O nosso agradecimento ao Fundo Social Europeu, ao Programa Operacional CENTRO 2020 e à Fundação para a Ciência e Tecnologia do Governo Português, instituiçóes públicas que se constituem como fontes de financiamento do Projeto de Investigação MAISaúde Mental - Monitorização e Avaliação dos Indicadores de Saúde Mental das crianças e adolescentes: da investigação à prática (código de identificação:CENTRO-01-0145-FEDER-023293), onde este estudo se integra.

\section{Colaborações}

Bica I, L Pinho MD, Silva EMB, Aparício G, Duarte J, Costa J e Albuquerque C contribuíram com a concepção do estudo, análise e interpretação dos dados, redação do artigo, revisão crítica relevante do conteúdo intelectual e aprovação da versão final a ser publicada.

\section{Referências}

1. Gaspar T, Matos MG. Qualidade de vida em crianças e adolescentes: Versão portuguesa dos instrumentos KidScreen. Lisboa: Fundação para a Ciência e Tecnologia, Faculdade de Motricidade Humana; 2008.

2. World Health Organization. Situation of child and adolescent health in Europe [Internet]. Copenhagen, Denmark: WHO Regional Office for Europe; 2018. [citado 2019 May 25]. Disponível em: http://www. euro.who.int/_data/assets/pdf_file/0007/381139/situation-childadolescent-health-eng.pdf?ua=1
3. Direcção Geral da Saúde (PT). Bases do programa nacional de saúde dos jovens. Lisboa: Direção Geral de Saúde: Divisão Materna, Infantil e dos Adolescentes; 2005.

4. Direcção Geral da Saúde (PT). Programa Nacional de Saúde dos Jovens 2006/2010. Direção Geral de Saúde: Divisão Materna, Infantil e dos Adolescentes; 2006

5. World Health Organization (WHO). Strategy for mental health and substance abuse in the Eastern Mediterranean Region 2012-2016. Technical paper [Internet] 2011 [2019 May 25]. Regional Committee for the Eastern Mediterranean, Fifty-eighth Session: World Health Organization. Disponível em: http://applications.emro.who.int/docs/ RC_technical_papers_2011_5_14223.pdf?ua=1

6. Gaspar T, Matos MG, Ribeiro JL, Leal I. Qualidade de vida e bem-estar em crianças e adolescentes. Rev Bras Ter Cogn. 2006;2(2):47-60.

7. Gaspar T, Cerqueira A, Branquinho C, Gaspar MM. Dimensions of social and personal skills in children and adolescents: age and gender differences. Int J Develop Res. 2018;8(1):18394-400.

8. Gaspar M. EquipaAventura Social. A saúde dos adolescentes portugueses após a recessão. Relatório do estudo HBSC 2018. [Internet] 2018 [citado 2019 May 25]. Equipa Aventura Social, Faculdade de Motricidade Humana, Universidade de Lisboa. Disponível em: http://aventurasocial. com/publicacoes/publicacao_1545534554.pdf

9. Agathão BT, Reichenheim ME, Moraes CL. Qualidade de vida relacionada à saúde de adolescentes escolares. Cien Saude Colet. 2018;23(2):659-68.

10. Viana C. Aumenta o fosso entre rapazes e meninas no sucesso escolar. Jornal Público [Internet] 2018 [citado 2019 May 25]. Disponível em: https://www.google.com/ search? client $=$ avast $\& q=8 .+$ Viana $+C .+$ Aumenta $+0+$ fosso + entre+rapazes+e+meninas+no+sucesso+escolar

11. Marcotte D, Fortin L, Potvin P, Papillon M. Gender differences in depressive symptoms during adolescence: role of gender-typed characteristics, self-esteem, body image, stressful life events, and pubertal status. J Emot Behav Disord. 2002;10(1):29-42.

12. Sweeting HN, West PB, Der GJ. Explanations for female excess psychosomatic symptoms in adolescence: evidence from a schoolbased cohort in the West of Scotland. BMC Public Health. 2007;7(1):298.

13. Jiménez-Iglesias A, Moreno C, Ramos P, Rivera F. What family dimensions are important for health-related quality of life in adolescence? J Youth Stud. 2015;18(1):53-67.

14. Santosa A, Kriegerb FV. Transtornos de humor na infância e na adolescência: uma actualização. Rev Bras Psicoter. 2014;16(1):104-14.

15. Borges A, Matos MG, Diniz JA. Estatuto familiar e autopercepção de saúde nos adolescentes. Temas Psicol. 2011;19(2):347-60.

16. Gaspar T, Ribeiro JL, Matos MG, Leal I. Promoção e qualidade de vida em crianças e adolescentes. Psicol Saude Doenças. 2008;9(1):55-71.

17. Berman AH, Liu B, Ullman S, Jadbäck I, Engström K. Children's quality of life based on the KIDSCREEN-27: child self-report, parent ratings and child-parent agreement in a Swedish random population sample. PLoS One. 2016;11(3):e0150545.

18. Analitis F, Velderman MK, Ravens-Sieberer U, Detmar S, Erhart M, Herdman M, et al.; European Kidscreen Group. Being bullied: associated factors in children and adolescents 8 to 18 years old in 11 European countries. Pediatrics. 2009;123(2):569-77.

19. Maria A, Guimarães C, Candeias I, Almeida S, Figueiredo C, Pinheiro $A$, et al. Qualidade de vida relacionada com a saúde em crianças e adolescentes portugueses: estudo numa população escolar. Acta Ped Portuguesa. 2017;48(3):203-11. 